\title{
THE REPRESENTATION ZETA FUNCTION OF A FAB COMPACT $p$-ADIC LIE GROUP VANISHES AT -2
}

\author{
JON GONZÁLEZ-SÁNCHEZ, ANDREI JAIKIN-ZAPIRAIN, \\ AND BENJAMIN KLOPSCH
}

\begin{abstract}
Let $G$ by compact $p$-adic Lie group and suppose that $G$ is FAb, i.e., that $H /[H, H]$ is finite for every open subgroup $H$ of $G$. The representation zeta function $\zeta_{G}(s)=\sum_{\chi \in \operatorname{Irr}(G)} \chi(1)^{-s}$ encodes the distribution of continuous irreducible complex characters of $G$. For $p \geq 3$ it is known that $\zeta_{G}(s)$ defines a meromorphic function on $\mathbb{C}$.

Wedderburn's structure theorem for semisimple algebras implies that $\zeta_{G}(-2)=|G|$ for finite $G$. We complement this classic result by proving that $\zeta_{G}(-2)=0$ for infinite $G$, assuming $p \geq 3$.
\end{abstract}

\section{INTRODUCTION}

Let $G$ be a finitely generated profinite group, and let $\operatorname{Irr}(G)$ denote the collection of all continuous irreducible complex characters of $G$. We observe that each $\chi \in \operatorname{Irr}(G)$ has finite degree and for every positive integer $n \in \mathbb{N}$ we put $r_{n}(G)=|\{\chi \in \operatorname{Irr}(G) \mid \chi(1)=n\}|$. From Jordan's theorem on finite linear groups in characteristic 0 (see [12, Theorem 9.2]) one deduces that $r_{n}(G)$ is finite for all $n \in \mathbb{N}$ if and only if $G$ is FAb, i.e., if $H /[H, H]$ is finite for every open subgroup $H$ of $G$.

Suppose that $G$ is FAb. Then the arithmetic sequence $r_{n}(G), n \in \mathbb{N}$, is encoded in the Dirichlet generating function

$$
\zeta_{G}(s)=\sum_{n=1}^{\infty} r_{n}(G) n^{-s}=\sum_{\chi \in \operatorname{Irr}(G)} \chi(1)^{-s}
$$

which is known as the representation zeta function of $G$. If the representation growth of $G$ is polynomially bounded, i.e., if $\sum_{n=1}^{N} r_{n}(G)=O\left(N^{d}\right)$ for some constant $d$, then $\zeta_{G}(s)$ defines an analytic function on a non-empty right half-plane of $\mathbb{C}$. Under favourable circumstances, this function admits a meromorphic continuation, possibly to the entire complex plane $\mathbb{C}$.

In recent years representation growth and representation zeta functions have been investigated for various kinds of groups, including compact $p$-adic Lie groups; for instance, see [6, 9, 1, 2] or the short introductory survey [7]. An intriguing, but mostly unexplored aspect is the significance of special

2010 Mathematics Subject Classification. 20E18 (11M41, 20C15, 22E50).

Key words and phrases. Representation zeta function, zeros, Brauer's Problem.

A. Jaikin-Zapirain and J. González-Sánchez were supported by grant MTM2011-28229C02-01 from the Spanish Ministry of Economy and Competitivity. J. González-Sánchez also acknowledges support through the Ramón y Cajal Programme of the Spanish Ministry of Science and Innovation. B. Klopsch is grateful for financial support which he received as a visitor at the Universidad Autónoma de Madrid. 
values of representation zeta functions. In particular, one may be curious about the locations of zeros and poles. While there is some theoretical understanding of the latter (see [2, Theorem B], and also compare [4] for the pole spectra of related zeta functions), almost nothing is known about the former.

In the present paper we establish that $\zeta_{G}(s)$ vanishes at $s=-2$ for every member $G$ of a certain class of profinite groups, including all infinite FAb compact $p$-adic Lie groups for $p \geq 3$. Indeed, let $G$ be a finitely generated profinite group which is FAb and virtually pro- $p$ for some prime $p$. We say that $G$ has rational representation zeta function (with respect to $p$ ), r.r.z.f. $(p)$ for short, if there exist finitely many positive integers $m_{1}, \ldots, m_{k}$ and rational functions $R_{1}, \ldots, R_{k} \in \mathbb{Q}(X)$ such that

$$
\zeta_{G}(s)=\sum_{i=1}^{k} m_{i}^{-s} R_{i}\left(p^{-s}\right) .
$$

In [6], Jaikin-Zapirain proved that, for $p \geq 3$, every FAb compact $p$-adic Lie group has r.r.z.f. $(p)$. It is conjectured that the result extends to 2-adic Lie groups; presently, it is known that every uniformly powerful pro-2 group has r.r.z.f. $(2)$.

There is only a small number of FAb compact $p$-adic Lie groups $G$ for which the representation zeta function $\zeta_{G}(s)$ has been computed explicitly; see [6, 1, 2]. By inspection of the formula given in [6, Theorem 7.5], Motoaki Kurokawa and Nobushinge Kurokawa noticed that the representation zeta function of the $p$-adic Lie group $\mathrm{SL}_{2}\left(\mathbb{Z}_{p}\right)$ has zeros at $s=-1$ and $s=-2$. The purpose of the present paper is to explain the zero at $s=-2$ which reflects a more general phenomenon.

Theorem 1. Let $G$ be a FAb profinite group which is infinite and virtually a pro-p group. If $G$ has rational representation zeta function with respect to $p$ then $\zeta_{G}(-2)=0$.

As indicated, using [6, Theorem 1.1] we derive the following corollary.

Corollary 2. Let $G$ be a FAb compact p-adic Lie group and suppose that $p \geq 3$. If $G$ is infinite then $\zeta_{G}(-2)=0$.

Remark 3. Wedderburn's structure theorem for semisimple algebras implies that $\zeta_{G}(-2)=\sum_{\chi \in \operatorname{Irr}(G)} \chi(1)^{2}=|G|$ for every finite group $G$. For an infinite profinite group $G$ one can evaluate $\zeta_{G}(s)$ at $s=-2$ only if the function defined by the Dirichlet series has a suitable continuation.

Remark 4 . The representation functions of compact open subgroups of semisimple $p$-adic Lie groups, such as $\mathrm{SL}_{n}\left(\mathbb{Z}_{p}\right)$, occur naturally as factors in Euler products for the representation zeta functions of arithmetic lattices in semisimple groups, such $\Gamma=\mathrm{SL}_{n}(\mathbb{Z}) \subseteq \mathrm{SL}_{n}(\mathbb{R})$; see [9, Proposition 1.3]. However, since the Euler product formula is not valid for $s=-2$, one cannot use Corollary 2 directly to investigate potential properties of $\zeta_{\Gamma}(s)$ at $s=-2$. For instance, the inverse of the Riemann zeta function $\zeta(s)^{-1}=$ $\sum_{n=1}^{\infty} \mu(n) n^{-s}$ satisfies $\zeta(s)^{-1}=\prod_{p}\left(1-p^{-s}\right)$ and $\zeta(0)=-2$.

In the next section we prove Theorem 1 and its corollary, by considering the $p$-adic limit of $\zeta_{G}(s)$ at $s=-2$. We also offer an alternative proof of 
Corollary 2, which is closer to the character theoretic set-up in [6]. In the last section we provide further comments and highlight some open problems.

In conclusion, we remark that related questions regarding zeros and special values of Witten $L$-functions associated to real Lie groups, in particular to the groups $\mathrm{SU}(2)$ and $\mathrm{SU}(3)$, have been considered by Kurokawa and Ochiai [8] and also by Min [10].

\section{THE PROOFS}

Proof of Theorem 1. Let $m_{1}, \ldots, m_{k} \in \mathbb{N}$ and $R_{1}, \ldots, R_{k} \in \mathbb{Q}(X)$ such that the Dirichlet series $\zeta_{G}(s)=\sum_{n=1}^{\infty} r_{n}(G) n^{-s}=\sum_{\chi \in \operatorname{Irr}(G)} \chi(1)^{-s}$ satisfies (1). Then the degrees of the irreducible characters of $G$ are of the form $m_{i} p^{r}$ with $i \in\{1, \ldots, k\}$ and $r \geq 0$. In particular, for every positive integer $j$ there are at most finitely many characters $\chi \in \operatorname{Irr}(G)$ with $p^{j} \nmid \chi(1)$. Consequently, the series $\zeta_{G}(s)$ converges, with respect to the $p$-adic topology, at every negative integer $-e \in-\mathbb{N}$ to an element in the ring $\mathbb{Z}_{p}$ of $p$-adic integers: we obtain a function

$$
\zeta_{G}^{p \text {-adic }}:-\mathbb{N} \rightarrow \mathbb{Z}_{p}, \quad-e \mapsto \sum_{n=1}^{\infty} r_{n}(G) n^{e}=\sum_{\chi \in \operatorname{Irr}(G)} \chi(1)^{e} .
$$

For the last equality recall that in the $p$-adic topology every converging series converges unconditionally so that its summands can be re-arranged freely.

Equation (11) reflects more than the equality of two complex functions: by expansion of the right hand side we obtain a Dirichlet series whose coefficients must agree with the defining coefficients $r_{n}(G)$ of the zeta function on the left hand side. This implies that for every negative integer $-e$,

$$
\zeta_{G}^{p \text {-adic }}(-e)=\sum_{i=1}^{k} m_{i}^{e} R_{i}\left(p^{e}\right)=\zeta_{G}(-e)
$$

Consequently, it suffices to prove that $\zeta_{G}^{p \text {-adic }}(-2)=0$.

Fix a positive integer $j$. As seen above, there are only finitely many characters $\chi \in \operatorname{Irr}(G)$ such that $p^{j} \nmid \chi(1)$. We define

$$
N_{j}=\bigcap_{\chi \in \operatorname{Irr}(G), p^{j} \nmid \chi(1)} \operatorname{ker} \chi
$$

where each ker $\chi$ coincides with the kernel of a representation affording $\chi$. Then $N_{j}$ is an open normal subgroup of $G$, and

$$
\zeta_{G}^{p \text {-adic }}(-2)=\sum_{\chi \in \operatorname{Irr}(G)} \chi(1)^{2}=\sum_{\substack{\chi \in \operatorname{Irr}(G) \text { with } \\ N_{j} \subseteq \operatorname{ker} \chi}} \chi(1)^{2}+\sum_{\substack{\chi \in \operatorname{Irr}(G) \text { with } \\ N_{j} \nsubseteq \operatorname{ker} \chi}} \chi(1)^{2} .
$$

The first sum is equal to the order of the finite group $G / N_{j}$, while all the terms in the second sum are divisible by $p^{2 j}$. Thus

$$
\zeta_{G}^{p \text {-adic }}(-2)=\left|G: N_{j}\right|+p^{2 j} a_{j},
$$

for some $a_{j} \in \mathbb{Z}_{p}$.

Since $G$ is infinite and virtually a pro- $p$ group, $\left|G: N_{j}\right|+p^{2 j} a_{j} \rightarrow 0$ in the $p$-adic topology as $j \rightarrow \infty$. Thus (3) yields $\zeta_{G}^{p \text {-adic }}(-2)=0$. 
Next we give an alternative proof of Corollary 2, which is closer to the set-up in [6] and does not rely on $p$-adic limits.

Proposition 5. Suppose that $p \geq 3$ and let $N$ be a FAb uniformly powerful pro-p group. Then for every $m \geq 0$,

$$
\zeta_{N^{p^{m}}}(s)=\left|N: N^{p^{m}}\right| \zeta_{N}(s) .
$$

Proof. This is a consequence of the analysis in [2, Section 3] of a formula given in [6, Corollary 2.13].

Proposition 6. Suppose that $p \geq 3$ and let $G$ be a FAb compact p-adic Lie group. Let $H$ be an open subgroup of $G$. Then

$$
\zeta_{G}(-2)=|G: H| \zeta_{H}(-2) .
$$

Proof. Choose an open normal subgroup $N$ of $G$ which is 2-uniform (in the sense of [6, Section 2]) and contained in $H$. We show that

$$
\zeta_{G}(-2)=|G: N| \zeta_{N}(-2) \text {. }
$$

The same reasoning yields $\zeta_{H}(-2)=|H: N| \zeta_{N}(-2)$, and combining the two equations proves the proposition.

We adapt the set-up in [6, Sections 5 and 6]. As in the proof of [6. Theorem 1.1], we decompose the representation zeta function of $G$ as

$$
\zeta_{G}(s)=\sum_{N \leq K \leq G} \sum_{\substack{\vartheta \in \operatorname{Irr}(N) \\ \text { with } \operatorname{St}_{G}(\vartheta)=K}}|G: K|^{-1-s} f_{(K, N, \vartheta)}(s) \cdot \vartheta(s)^{-1},
$$

where for each character triple $(K, N, \vartheta)$ one defines

$$
f_{(K, N, \vartheta)}(s)=\sum_{\chi \in \operatorname{Irr}(K \mid \vartheta)}\left(\frac{\chi(1)}{\vartheta(1)}\right)^{-s}
$$

summing over all $\chi \in \operatorname{Irr}(K)$ such that $\vartheta$ is a component of $\operatorname{red}_{N}^{G}(\chi)$. We observe that for each character triple $(K, N, \vartheta)$,

$$
f_{(K, N, \vartheta)}(-2)=\sum_{\chi \in \operatorname{Irr}(K \mid \vartheta)}\left(\frac{\chi(1)}{\vartheta(1)}\right)^{2}=\frac{\operatorname{red}_{N}^{K}\left(\operatorname{ind}_{N}^{K}(\vartheta)\right)(1)}{\vartheta(1)}=|K: N| .
$$

It is proved in [6] that for each group $K$ with $N \leq K \leq G$ the set $\operatorname{Irr}(N)_{K}=\left\{\vartheta \in \operatorname{Irr}(N) \mid \operatorname{St}_{G}(\vartheta)=K\right\}$ can be partitioned into finitely many subsets $\operatorname{Irr}(N)_{K, v}$, labelled by $v \in V_{K}$, such that

(i) for each $v \in V_{K}$ and $\vartheta \in \operatorname{Irr}(N)_{K, v}$,

$$
f_{(K, N, \vartheta)}(s)=f_{v}(s)
$$

depends only on $v$ and

(ii) for each $v \in V_{K}$,

$$
g_{v}(s)=\sum_{\vartheta \in \operatorname{Irr}(N)_{K, v}} \vartheta(s)^{-1}
$$

is a rational function over $\mathbb{Q}$ in $p^{-s}$. 
The equations

$$
\begin{aligned}
\zeta_{G}(s) & =\sum_{N \leq K \leq G} \sum_{v \in V_{K}}|G: K|^{-1-s} f_{v}(s) g_{v}(s), \\
\zeta_{N}(s) & =\sum_{N \leq K \leq G} \sum_{v \in V_{K}} g_{v}(s)
\end{aligned}
$$

combined with (4) give

$$
\begin{aligned}
\zeta_{G}(-2) & =\sum_{N \leq K \leq G} \sum_{v \in V_{K}}|G: K||K: N| g_{v}(-2) \\
& =|G: N| \zeta_{N}(-2) .
\end{aligned}
$$

Second proof of Corollary 2. By Proposition 6 it is enough to prove the result for a uniformly powerful pro- $p$ group $N$. By Propositions 5 and 6 we have

$$
\left|N: N^{p}\right| \zeta_{N}(-2)=\zeta_{N^{p}}(-2)=\left|N: N^{p}\right|^{-1} \zeta_{N}(-2) .
$$

Since $\left|N: N^{p}\right|>1$, this implies $\zeta_{N}(-2)=0$.

\section{Open questions}

We highlight three questions which arise naturally from Theorem 1, Corollary 2 and their proofs.

In view of (2) we record the following problem.

Question 1. Let $G$ be a FAb compact $p$-adic analytic group. What are the values of $\zeta_{G}(s)$ at other negative integers $s=-e$ and is there a suitable interpretation of these?

Of course, we would like to extend Corollary 2 to the prime $p=2$. More generally, one can ask the following.

Question 2. Let $G$ be a FAb profinite group and and suppose that $\zeta_{G}(s)$ converges in some right half-plane $\{s \in \mathbb{C} \mid \operatorname{Re}(s)>\alpha\}$. Suppose further that $\zeta_{G}(s)$ has a meromorphic continuation so that $\zeta_{G}(-2)$ is defined. Is it true that $\zeta_{G}(-2)=0$ ?

For instance it would be natural to investigate this question for compact analytic groups over compact discrete valuation rings of positive characteristic, e.g., over $\mathbb{F}_{p} \llbracket t \rrbracket$. The representation zeta functions of such groups are still rather poorly understood. In particular, no analogue of Proposition 5 is known. However, a direct computation in [6] shows that, for $p \geq 3$, the group $\mathrm{SL}_{2}\left(\mathbb{F}_{p} \llbracket t \rrbracket\right)$ has the same representation zeta function as the $p$-adic analytic group $\mathrm{SL}_{2}\left(\mathbb{Z}_{p}\right)$.

The last question is inspired by Brauer's Problem 1, which asks: what are the possible degree patterns for irreducible characters of finite groups; see [3, 5, 11]. Given a profinite group $G$ with r.r.z.f. ${ }_{(p)}$, the completed group algebra $\mathbb{C} \llbracket G \rrbracket=\lim _{N \unlhd G} \mathbb{C}[G / N]$, formed with respect to the directed set of normal open subgroups of $G$, determines the representation zeta function of $G$ and, conversely, $\zeta_{G}(s)$ determines $\mathbb{C} \llbracket G \rrbracket$. Furthermore, if $G$ is a pro- $p$ group, then $\zeta_{G}(s)$ is a rational function over $\mathbb{Q}$ in $p^{-s}$. The following can be regarded as an extension of Brauer's Problem 1 to FAb pro- $p$ groups. 
Question 3. Which rational functions $R\left(p^{-s}\right)$ over $\mathbb{Q}$ in $p^{-s}$ are representation zeta functions of infinite FAb pro- $p$ groups with r.r.z.f. ${ }_{(p)}$ ?

Theorem 1 provides a first necessary criterion: $R\left(p^{2}\right)=0$.

\section{REFERENCES}

[1] N. Avni, B. Klopsch, U. Onn, C. Voll, Representation zeta functions of some compact p-adic analytic groups, in: Zeta Functions in Algebra and Geometry (Contemporary Mathematics 566, Amer. Math. Soc., Providence, RI, 2012), 295-330.

[2] N. Avni, B. Klopsch, U. Onn, C. Voll, Representation zeta functions of compact p-adic analytic groups and arithmetic groups, Duke Math. J. 162 (2013), 111-197.

[3] R. Brauer, Representations of finite groups, in: Lectures on Modern Mathematics, Vol. I (Wiley, New York, 1963), 133-175.

[4] M. du Sautoy, Zeta functions of groups and rings: uniformity Israel J. Math. 86 (1994), 1-23.

[5] B. Huppert, Research in representation theory at Mainz (1984-1990), in: Representation theory of finite groups and finite-dimensional algebras (Progr. Math. 95, Birkhäuser, Basel, 1991), 17-36.

[6] A. Jaikin-Zapirain, Zeta function of representations of compact p-adic analytic groups, J. Amer. Math. Soc. 19 (2006), 91-118.

[7] B. Klopsch, Representation growth and representation zeta functions of groups, to appear in Note Mat. 33 (2013).

[8] N. Kurokawa and H. Ochiai, Zeros of Witten zeta functions and absolute limit, preprint.

[9] M. Larsen and A. Lubotzky, Representation growth of linear groups, J. Eur. Math. Soc. 10 (2008), 351-390.

[10] J. Min, Zeros and special values of Witten zeta functions and Witten L-functions, preprint.

[11] A. Moreto, Complex group algebras of finite groups: Brauer's problem 1, Adv. Math. 208 (2007), 236-248.

[12] B. A. F. Wehrfritz, Infinite linear groups, Ergebnisse der Matematik und ihrer Grenzgebiete 76, Springer-Verlag, New York-Heidelberg, 1973.

Departamento de Matemáticas, Facultad de Ciencia y Tecnología, UniverSidad del País Vasco- Euskal Herriko Unibertsitatea, Apartado 64448080 BilBAO, SPAIN

E-mail address: jon.gonzalez@ehu.es

Departamento de Matemáticas, Facultad de Ciencias, módulo 17, Universidad Autónoma de Madrid, Ciudad Universitaria de Cantoblanco, 28049-Madrid, SPAIN

E-mail address: andrei.jaikin@uam.es

Institut für Algebra und Geometrie, Mathematische Fakultät, Otto-vonGuericke-Universität Magdeburg, 39106 Magdeburg, Germany

E-mail address: Benjamin.Klopsch@ovgu.de 